\title{
Imaging of Juvenile Rheumatoid Arthritis
}

\author{
Carolyn M. Sofka, MD • Eric Bogner, MD
}

Received: 7 November 2007/Accepted: 7 November 2007/Published online: 14 December 2007

(C) Hospital for Special Surgery 2007

\section{Introduction}

Juvenile rheumatoid arthritis (JRA) is a chronic, often debilitating disorder with multisystem involvement. Detailed imaging of JRA is needed for initial diagnosis, monitoring of disease activity, and determining extraarticular and other complications [1]. We present a case of a patient with chronic JRA and neck pain in which multiple imaging modalities were employed.

\section{Case report}

This is a 40-year-old female with a longstanding history of JRA presenting at this time for magnetic resonance imaging (MRI) of the cervical spine with neck pain and increasing numbness in the fingers. MRI of the cervical spine demonstrated the characteristic changes of an inflammatory arthritis with fusion of the vertebral bodies and posterior elements in the cervical and thoracic spines (Fig. 1). In addition, at C5-6, there was a broad-based, left-sided disc protrusion with severe central canal stenosis and abnormal cord signal (Fig. 2). Subsequent radiographs of the cervical spine again demonstrated multilevel fusion in the cervical spine, in addition to severe arthrosis at the $\mathrm{C} 1-2$ level (Fig. 3).

The patient was then referred for computed tomographic (CT) myelogaphy of the spine for additional evaluation and presurgical planning. Thin-cut, high-resolution CT images of the cervical spine obtained after the introduction of intrathecal contrast material demonstrated moderately extensive bony proliferation, in addition to the disc pathology, contributing to the central canal stenosis at the C5-6 level with mild impingement and flattening of the cord (Fig. 4).

C. M. Sofka, MD $(\bowtie) \cdot$ E. Bogner, MD

Department of Radiology and Imaging,

Hospital for Special Surgery,

535 East 70th Street, New York, NY 10021, USA

e-mail: sofkac@hss.edu

\section{Discussion}

Many imaging modalities are useful for imaging patients with inflammatory arthropathies. Radiographs remain the mainstay of diagnostic imaging, although their sensitivity for diagnosing erosions and soft tissue and periarticular involvement of disease is limited [2]. Radiographs, however, do play a central role in the evaluation of dynamic instability (atlantoaxial subluxation) in rheumatoid arthritis [3]. Cross-sectional imaging, such as MRI, CT, and ultrasound, has improved sensitivity to visualize the soft tissues (MRI, ultrasound) and the osseous structures (MRI, CT).

CT provides exquisite detail of cortical bone, clearly demonstrating the presence of cortical erosions in the small joints of the hands, feet, and sacroiliac joints, an area traditionally difficult to image with conventional radiographs, as well as ankylosis of the apophyseal joints of the cervical spine $[4,5]$. The tomographic nature of CT and MRI allows for imaging the axial and appendicular skeleton in multiple planes, which is especially useful when imaging the spine.

The superior soft-tissue contrast of MRI and ultrasound allows for detailed evaluation of the soft tissues. The ability to directly visualize synovial inflammation, regional tendon pathology (tenosynovitis), and cortical erosions allows for earlier diagnosis of disease and joint involvement and destruction [6-8] (Fig. 5).

The ability of MRI and ultrasound to detect early, often subclinical disease by directly visualizing synovial inflammation is an advantage with regards to the prompt initiation of disease treatment [2, 9-11]. Both CT and fluoroscopy can also be used to guide for therapeutic interventions, as well as diagnostic procedures, such as myelography, providing additional dynamic and functional information. The real-time capabilities of ultrasound also result in its ability to guide for procedures, such as fluid aspirations and therapeutic injections, with the additional applications of power Doppler often providing a semiquantitative monitoring response to treatment [11, 12].

In summary, multiple imaging modalities play a role in the evaluation of patients with inflammatory arthropathies such as JRA. Radiographs remain the initial method of 


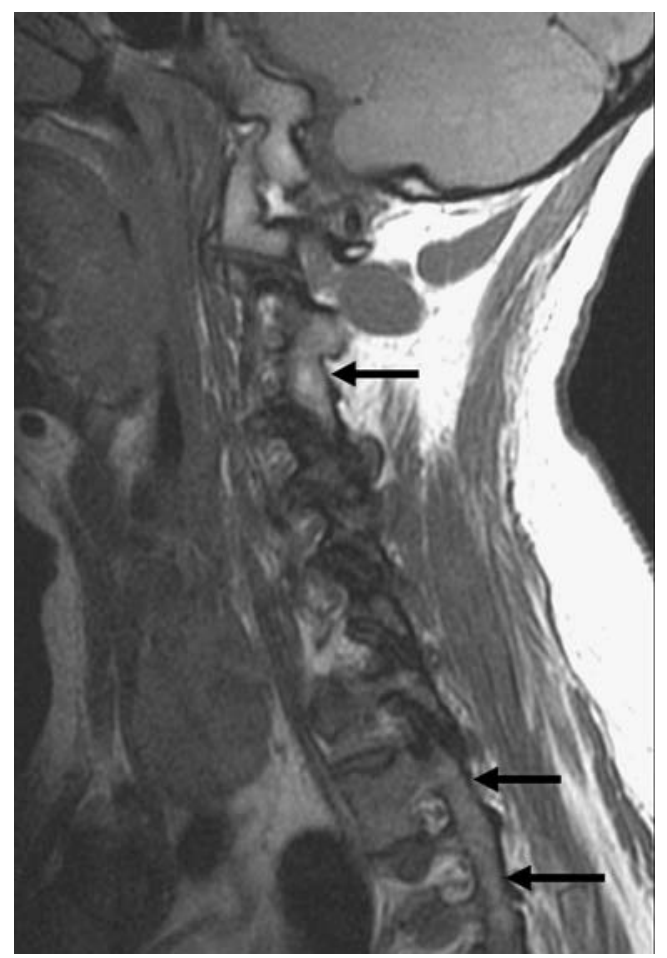

Fig. 1. Parasagittal $T 1$ weighted $M R$ image of the cervical spine demonstrating fusion of the upper cervical spine and upper thoracic spine vertebral bodies and posterior elements (arrows) consistent with history of chronic JRA

evaluation and have utility in diagnosing subluxations of the spine with flexion and extension views. CT provides exquisite detail of osseous anatomy and cortical bone, being extremely sensitive to the diagnosis of cortical erosions and inflammatory changes in the spine. MRI and ultrasound can directly image the soft tissues, often detecting early, subclinical disease and synovitis and inflammation, thus initiating earlier treatment. Ultrasound

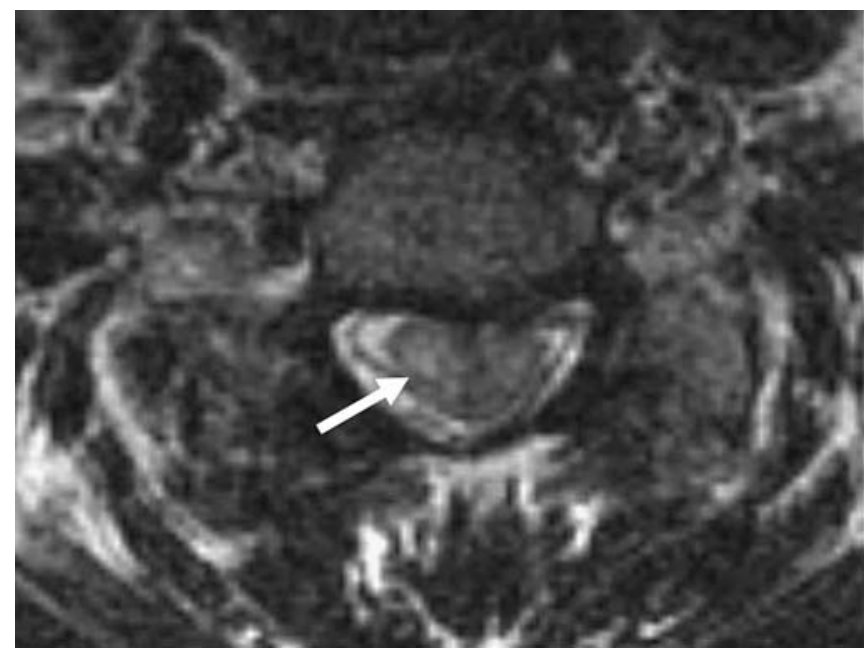

Fig. 2. Axial T2 weighted MR image at the C5-6 level demonstrating central canal stenosis and abnormal cord signal (arrow)

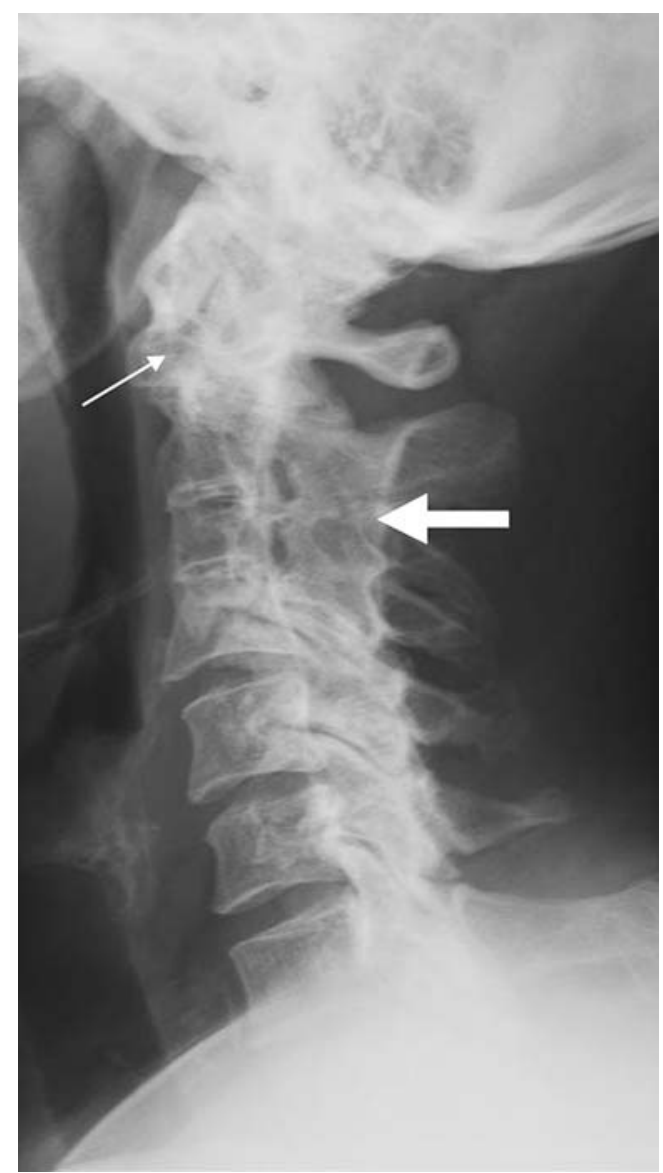

Fig. 3. Lateral radiograph of the cervical spine demonstrating fusion of the vertebral bodies and posterior elements in the upper cervical spine (thick white arrow) and arthritic changes at the $\mathrm{C} 1-2$ articulation (thin white arrow)

further can guide for therapeutic injections and provide a semiquantitative method of following disease activity and progression. The complementary nature of all imaging modalities results in a comprehensive evaluation of the patient with JRA.

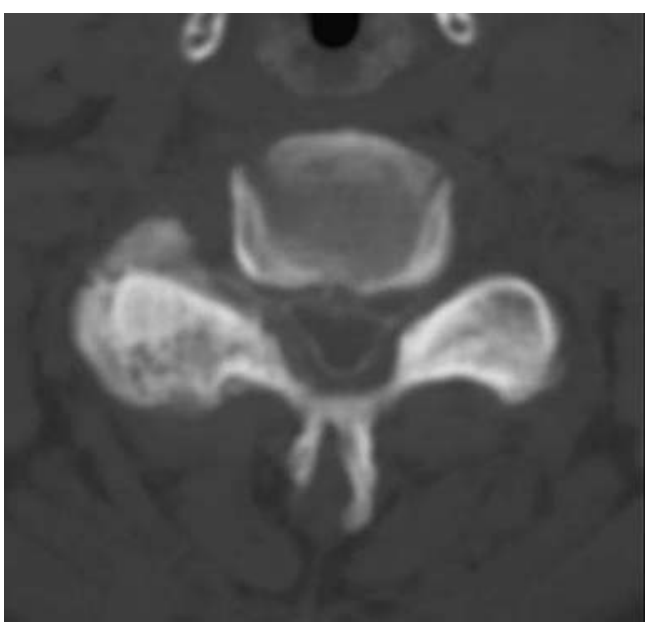

Fig. 4. Axial image from CT myelogram at the C5-6 level demonstrating central canal stenosis with only a very thin band of intrathecal contrast material extending around the cord 


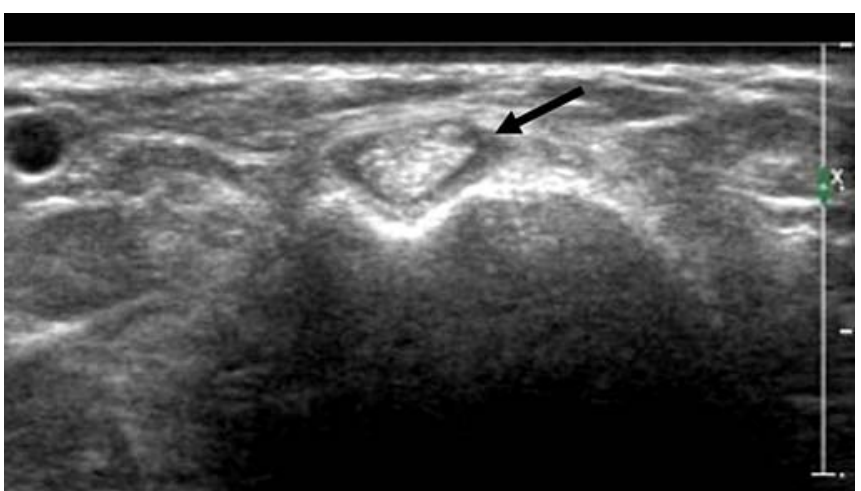

Fig. 5. Short axis ultrasound image of the wrist in the same patient several months prior demonstrating hypoechoic thickening of the tendon sheath (arrow)

\section{References}

1. Johnson K (2006) Imaging of juvenile idiopathic arthritis. Pediatr Radiol 36(8)743-758

2. Doria AS, Kiss MH, Lotito AP, Molnar LJ, de Castro CC, Medeiros CC, Cerri GG (2001) Juvenile rheumatoid arthritis of the knee: evaluation with contrast-enhanced color Doppler ultrasound. Pediatr Radiol 31(7)524-531

3. Laiho K, Soini I, Kautiainen H, Kauppi M (2003) Can we rely on magnetic resonance imaging when evaluating unstable atlantoaxial subluxation. Ann Rheum Dis 62(3)254-256

4. Reiter MF, Boden SD (1998) Inflammatory disorders of the spine. Spine 23(24)2755-2766

5. Resnick D (1989) Inflammatory disorders of the vertebral column: seronegative spondyloarthopathies, adult-onset rheumatoid arthritis, and juvenile chronic arthritis. Clin Imaging 13(4)253-268

6. Frosch M, Foell D, Ganser G, Roth J (2003) Arthrosonography of hip and knee joints in the follow up of juvenile rheumatoid arthritis. Ann Rheum Dis 62(3)242-244

7. Johnson K, Wittkop B, Haigh F, Ryder C, Gardner-Medwin JM (2002) The early magnetic resonance imaging features of the knee in juvenile idiopathic arthritis. Clin Radiol 57(6)466-471

8. Bellah R (2001) Ultrasound in pediatric musculoskeletal disease: techniques and applications. Radiol Clin North Am 39(4)597-618

9. Gylys-Morin VM, Graham TB, Blebea JS, Dardzinski BJ, Laor T, Johnson ND, Oestreich AE, Passo MH (2001) Knee in early juvenile rheumatoid arthritis: MR imaging findings. Radiology 220(3)696-706

10. El-Miedany YM, Housny IH, Mansour HM, Mourad HG, Mehanna AM, Megeed MA (2001) Ultrasound versus MRI in the evaluation of juvenile idiopathic arthritis of the knee. Joint Bone Spine 68(3)222-230

11. Lamer S, Sebag GH (2000) MRI and ultrasound in children with juvenile chronic arthritis. Eur J Radiol 33(2)85-93

12. Eich GF, Halle F, Hodler J, Seger R, Willi UV (1994) Juvenile chronic arthritis: imaging of the knees and hips before and after intraarticular steroid injection. Pediatr Radiol 24(8)558-563 